\title{
Relação entre ações de qualidade de vida no trabalho e bem-estar laboral
}

\author{
Relation between quality of life at work and laboral well-being
}

Priscila Roriz Couto ${ }^{[a]}$, Tatiane Paschoal[ ${ }^{[b]}$

\footnotetext{
[a] Graduada em Psicologia pelo Instituto de Ensino Superior de Brasília (IESB), Brasília, DF - Brasil, e-mail: priroriz@ hotmail.com

${ }^{[b]}$ Doutora em Psicologia Social, do Trabalho e das Organizações pela Universidade de Brasília (UnB), professora do curso de Administração da Universidade de Brasília, Brasília, DF - Brasil.
}

Recebido: 29/01/2010 Received: 01/29/2010

Aprovado: 10/05/2011 Approved: 05/10/2011

\section{Resumo}

Graças às novas demandas do mundo laboral, as organizações têm buscado, cada vez mais, ações adequadas às necessidades de seus colaboradores. 0 presente estudo teve como objetivo investigar o impacto de atividades organizacionais voltadas à saúde do trabalhador sobre o bem-estar no trabalho. 0 bem-estar laboral foi definido como a prevalência de emoções positivas e a percepção do indivíduo de que, no seu trabalho, desenvolve seus potenciais e avança no alcance de suas metas de vida. Foram consideradas as seguintes ações voltadas à saúde: ginástica laboral, ambiente com musicalização, ambiente para relaxamento, automassagem e comemoração de aniversariantes. Participaram do estudo 130 trabalhadores de diferentes organizações do Distrito Federal. 0 bem-estar no trabalho foi medido pela EBET, instrumento já validado no Brasil, que operacionaliza o construto a partir de três fatores: afeto positivo, afeto negativo e realização pessoal no trabalho. Para avaliação das ações voltadas à saúde do trabalhador, foram desenvolvidos itens fechados específicos. Os dados foram analisados por meio de regressão múltipla stepwise, que considera apenas critérios estatísticos para a entrada das variáveis no modelo de predição. Verificou-se que quanto maior a frequência da participação em atividades como ginástica laboral, momentos de relaxamento, sala com musicalização, comemoração dos aniversariantes e automassagem, menor é o afeto negativo no trabalho. Afeto positivo e realização não foram preditos pelas ações de saúde. Os resultados são discutidos no texto e as limitações do estudo apontadas.

Palavras-chave: Qualidade de vida no trabalho. Felicidade no trabalho. Afeto positivo. Afeto negativo. Realização pessoal.

\section{Abstract}

Due to the new demands on the world of work, organizations have searched for appropriated practices to support their employees' needs. This study aimed to investigate the impact of organizational practices towards employee's health on laboral well-being. Laboral well-being was defined as the prevalence of positive emotions and the perception that one can develop his potentials and advance on his own goals in life at the workplace. The following health practices were considered: laboral gymnastics, music at work, location to relax, self-massage and birthday comemmorations. A total of 130 workers from different organizations in the Brazilian Federal District participated in the study. Laboral well-being construct was measured by WWS, a Brazilian validated 


\begin{abstract}
instrument which considers three factors: positive affect, negative affect and self achievement at work. Specific itens were developed assess the practices. Data were analized with the use of stewpwise multiple regression, which only involves statistic elements to find the prediction model. Results appointed that participation in activities such as laboral gymnastics, relaxing moments, music at work, birth comemmorations, and self-massage are the most frequent; the least frequent being negative affect experiences. Positive affect and achievement were not predicted by practices towards health-directed actions. Results and limitations of the study are respectively discussed and pointed out in the text.
\end{abstract}

Keywords: Quality of life at work. Work happiness. Positive affect. Negative affect. Self-achievement.

\section{Introdução}

Estresse, mau humor, cansaço, fadiga e até mesmo depressão são exemplos de sintomas comuns de pessoas que cumprem longas e cansativas jornadas de trabalho, ficam muito tempo sem tirar férias, ou não possuem uma folga para lidar com a rotina. Por essa razão, a maneira como as empresas lidam com seus colaboradores deve ser adequada às novas demandas do mundo laboral, para que, assim, haja melhorias na forma como os funcionários lidam com o trabalho e, consequentemente, com tarefas do seu dia a dia. O que pode ser observado é que, cada vez mais, as pessoas têm menos tempo para si mesmas (Vasconcelos, 2001). Portanto, pensar em qualidade de vida é pensar em uma constante, que deve ser valorizada em todos os lugares, e em especial no ambiente de trabalho, pois esse é o local onde muitas pessoas passam o maior tempo do seu dia.

Atualmente, algumas empresas estão buscando trabalhar com uma gestão focada na mudança e na transformação de processos políticos ou comportamentais, em seus diferentes graus de atividade (Carvalho, Leite, Rohm \& Oliveira, 2008). Existem três etapas importantes em um processo de gestão de mudança nas organizações: envolvimento, desenvolvimento e a internalização. Na etapa do envolvimento é fundamental a participação dos colaboradores. 0 envolvimento ocorre quando se percebe que o entendimento e o nível de motivação dos indivíduos passam para um estágio em que dificilmente poderá ocorrer regressão. 0 desenvolvimento consiste em descrever a situação real, identificar a situação desejada, definir a lacuna existente e utilizar o conhecimento evidenciado na etapa de envolvimento, para reduzi-la até alcançar o resultado esperado. Por fim, a internalização seria a utilização de práticas dos novos parâmetros e a visualização do que se conseguiu obter com ações diferenciadas. (Carvalho, Leite, Rohm \& Oliveira, 2008).

As mudanças nas organizações, cujos eventos estão presentes em quase todos os contextos de trabalho, podem trazer consequências negativas ou positivas para os colaboradores. 0 tipo de consequência depende de características pessoais e de práticas de gestão adotadas pela organização (Paschoal, 2008). Assim, a instituição deve se preocupar em focar na qualidade de vida e no bem-estar dos funcionários, desenvolvendo estratégias variadas, que compreendem desde mudanças estruturais até atividades mais simples, como ginásticas laborais, momentos de relaxamento ou orientação para uma alimentação mais equilibrada (Vasconcelos, 2001).

Alguns autores têm realizado pesquisas com o objetivo de compreender melhor o conceito da qualidade de vida e do bem-estar do funcionário em sua atividade laboral (França, 1997; Paschoal \& Tamayo, 2008; Vasconcelos, 2001). A compreensão de cada fenômeno, de seus limites conceituais e formas de operacionalização orientam tanto o desenvolvimento de pesquisas acadêmicas quanto atividades de diagnóstico e avaliações no contexto organizacional.

No que diz respeito à qualidade de vida no trabalho (QVT), ao longo dos anos, foram desenvolvidas diferentes propostas sobre o que constitui o fenômeno e como ele é operacionalizado nas organizações. Apesar das divergências, existe uma ideia predominante de que QVT envolve estratégias organizacionais (Vasconcelos, 2001). De acordo com Limongi-França (1997), os processos de QVT podem ser definidos como um 
conjunto de práticas ou ações organizacionais que envolvem a implementação de melhorias e inovações no ambiente de trabalho. 0 estabelecimento da QVT só terá andamento quando a empresa olhar seus colaboradores como um todo, o que é denominado de enfoque biopsicossocial. Tal perspectiva representa um fator diferencial para a realização de diagnósticos, campanhas, criação de serviços e implantação de projetos voltados para a preservação e desenvolvimento das pessoas nas empresas.

No enfoque biopsicossocial, a pessoa é o centro das análises e os fatores psicossociais são fundamentais para explicação da saúde e da doença na sociedade moderna (Limongi-França, 2009). A dimensão biológica da abordagem biopsicossocial refere-se às características físicas herdadas ou adquiridas ao nascer e durante toda a vida. A dimensão psicológica envolve processos afetivos, emocionais e cognitivos, que formam a personalidade da pessoa e seu modo de perceber as circunstâncias da vida. Por fim, a dimensão social revela os valores socioeconômicos, a cultura, as crenças e a organização dos grupos dos quais a pessoa participa. Segundo Limongi-França (2009), o enfoque biopsicossocial de QVT está de acordo com uma visão holística do homem e, nas organizações, as ações voltadas à saúde do trabalhador devem considerar sua subjetividade, multidimensionalidade, experiências positivas e negativas.

Segundo Ferreira (2006), as abordagens de estudo e gestão da QVT podem ser classificadas como assistencialista ou preventiva. A abordagem assistencialista responsabiliza o próprio trabalhador por sua QVT, defende o uso de atividades de natureza compensatória do desgaste que o trabalhador vivencia em função do trabalho e enfatiza resultados como o aumento da produtividade (Ferreira, Ferreira, Antloga \& Bergamaschi, 2009). O foco da abordagem preventiva, por sua vez, compreende a remoção de problemas geradores de mal-estar nas organizações. A QVT seria, portanto, concebida como uma tarefa de todos na organização, devendo haver uma busca permanente pela harmonia entre o bem-estar, a eficiência e a eficácia nos ambientes organizacionais (Ferreira, Ferreira, Antloga \& Bergamaschi, 2009).

Quando adota uma abordagem assistencialista, a organização geralmente oferece aos trabalhadores atividades como ginástica laboral, massagens, eventos sociais comemorativos, que constituem ações para minimização do estresse. Segundo Ferreira et al. (2009), em programas de QVT assistencialistas, o trabalhador é a variável de ajuste e não haveria necessidade de modificar seu contexto de produção. A abordagem preventiva tem sido defendida por muitos autores, pois considera a intervenção em condições e organização do trabalho, além do envolvimento mais amplo dos membros organizacionais. Apesar disso, muitos gestores acabam adotando, em seus programas, ações específicas contra o estresse e voltadas para a saúde do trabalhador (Ferreira, 2006; Ferreira et al., 2009).

Assim como a saúde do trabalhador, o bem-estar no trabalho consiste em um fenômeno presente nos discursos de gestores e acadêmicos que se referem a ações de QVT. Muitas vezes, o bem-estar é considerado o próprio objetivo dessas práticas organizacionais (Limongi-França, 2009). Mas, em que consiste o bem-estar no trabalho? De que forma é influenciado por ações de QVT? A seguir, é apresentada uma breve revisão desse conceito e de seus antecedentes.

\section{Bem-estar}

A revisão de literatura indica que não existe consenso sobre as definições de bem-estar. Contudo, o que pode ser evidenciado é que o bem-estar e a felicidade são termos que se misturam nas definições científicas. Segundo Alburquerque e Tróccoli (2004), existem duas correntes principais na área do bem-estar geral: bem-estar subjetivo e bem-estar psicológico. Essas duas correntes influenciam o estudo do bem-estar no trabalho.

0 bem-estar subjetivo tem atraído um enorme interesse por parte dos pesquisadores nos últimos anos e pode ser considerado como uma avaliação subjetiva da qualidade de vida (Albuquerque \& Tróccoli, 2004). Três dimensões operacionalizam o bem-estar subjetivo: afeto positivo, afeto negativo e satisfação com a vida. 0 bem-estar seria mais elevado quanto mais o afeto positivo prevalecesse sobre o afeto negativo e maior fosse a satisfação com a vida em geral. 0 bem-estar psicológico, por sua vez, pode ser entendido como a experiência de expressão pessoal e de autorrealização (Ryff, 1989; Waterman, 1993; Waterman, Schwartz \& Conti, 2008).

Segundo Ryff (1989), existem seis elementos que definem o bem-estar psicológico: autoaceitação, relação positiva com os outros, autonomia, controle do ambiente, propósito na vida e crescimento pessoal. Autoaceitação consiste em atitudes positivas do 
indivíduo em relação a si mesmo. Relação positiva com os outros é a satisfação e confiança na relação com os outros, pode ser definido como fortes sentimentos de empatia, afeição, amizade e identificação com o outro. Autonomia consiste na independência e autorregulação do comportamento. Controle do ambiente é a habilidade do indivíduo para escolher ou criar situações adequadas às condições pessoais. Propósito na vida é a manutenção de objetivos, intenções e de senso de direção que contribui para a percepção de que a vida é significativa. E por fim, o crescimento pessoal que consiste no desenvolvimento do potencial do indivíduo e da sua expansão como pessoa.

De uma forma geral, quando se trata do bem-estar no contexto do trabalho, os pesquisadores se apoiam em uma ou outra abordagem e utilizam diferentes conceitos, sejam relacionados a experiências positivas, como a satisfação no trabalho (Amaral \& Siqueira, 2004), ou variáveis relacionadas a experiências negativas, como o burnout (Maslach, Schaufeli \& Leiter, 2001). Este estudo se preocupa em enfatizar as experiências positivas do trabalhador. Além disso, segue uma tendência da literatura internacional em considerar tanto a dimensão hedônica e afetiva do bem-estar quanto uma dimensão de realização e expressão pessoal. Segundo Waterman (1993) e Waterman, Schwartz e Conti (2008), diferentes situações podem gerar experiências de bem-estar distintas e tanto as emoções prazerosas quanto a percepção de realização são fundamentais para a compreensão do complexo fenômeno da felicidade ou bem-estar pessoal.

Warr (2007) afirma que existem dois tipos importantes e complementares de bem-estar no trabalho. 0 primeiro corresponde a sentimentos de prazer vivenciados pelo trabalhador e se relaciona com a felicidade hedônica. 0 segundo tipo de bem-estar no trabalho refere-se a experiências denominadas de autovalidação. Quanto à felicidade hedônica, emoções de prazer e excitação combinam-se para determinar a tônica afetiva do indivíduo. Emoções de prazer, entusiasmo e conforto são representantes do afeto positivo e indicam alto bem-estar, enquanto emoções de desprazer, ansiedade e depressão representam o afeto negativo e indicam baixo bem-estar. Quanto à autovalidação, o desenvolvimento de atributos pessoais, a exploração do próprio potencial e a expressão pessoal do trabalhador são seus aspectos constituintes (Warr, 2007).
Neste estudo, o bem-estar no trabalho é entendido como a prevalência de emoções positivas e a percepção do indivíduo de que, no seu trabalho, é possível desenvolver seus potenciais e avançar no alcance de suas metas de vida (Paschoal \& Tamayo, 2008). Portanto, o bem-estar no trabalho inclui tanto aspectos afetivos (emoções e humores) quanto cognitivos (percepção de realização). Em termos operacionais, bem-estar no trabalho é concebido em torno de três fatores: afeto positivo, afeto negativo e realização pessoal no trabalho. Os dois primeiros referem-se aos elementos hedônicos do bem-estar no trabalho e o último, aos elementos relacionados à realização e à expressão pessoal (Paschoal \& Tamayo, 2008).

Uma preocupação dos gestores de pessoas consiste em identificar variáveis que influenciem o bem-estar no trabalho. E o que a literatura tem revelado a esse respeito? Alguns avanços já foram realizados em relação ao bem-estar geral, especialmente sobre o bem-estar subjetivo (Diener \& Suh, 1999). 0 número de estudos sobre antecedentes do bem-estar no trabalho está crescendo na medida em que modelos teóricos e empíricos começam a ganhar força na literatura organizacional.

Sobre a influência de variáveis pessoais, estudos revelam que existe relação entre afetividade negativa e elementos do bem-estar, como a ansiedade no trabalho (Jex \& Spector, 1996). Segundo Warr (2007), as variáveis pessoais não devem ter impacto direto sobre o bem-estar geral, mas devem interagir com diferentes variáveis situacionais. As condições de trabalho podem afetar o bem-estar principalmente quando atrapalham ou facilitam o avanço nas metas individuais. Tal questão necessita ser mais explorada empiricamente, mas levanta a possibilidade de variáveis pessoais serem mediadoras de aspectos do contexto que influenciam o bem-estar.

De forma geral, a literatura organizacional sugere que o bem-estar no trabalho seja determinado por variáveis situacionais relacionadas ao próprio trabalho. Características do contexto organizacional, como autonomia, ampliação de conhecimentos relacionados ao trabalho, conflitos entre papéis, ambiguidade de papéis, suporte social, padrão afetivo do grupo, oportunidades de desenvolvimento na carreira e percepção de justiça, foram associadas a algum aspecto do bem-estar por meio de estudos empíricos (Warr, 2007). 
Existem algumas lacunas que necessitam ser preenchidas no campo do bem-estar. A revisão de literatura sobre antecedentes do bem-estar no trabalho revelou a existência de estudos que abordam as emoções e humores no trabalho (Barsky \& Kaplan, 2007; Weiss, Suckow \& Cropanzano, 1999), que consistem na dimensão hedônica do bem-estar. Apesar disso, muitos autores enfocam emoções negativas, como ansiedade, tensão e depressão (Strazdins, D’Souza, Lim, Broom \& Rodgers, 2004). A dimensão referente à realização e à expressão pessoal do bem-estar no trabalho, por sua vez, não costuma ser incluída nas pesquisas e, quando abordada, os autores fazem-no indiretamente, por meio de alguma variável relacionada, como a percepção de sucesso no trabalho (Wiese \& Freund, 2005).

A literatura da qualidade de vida tende a considerar variáveis como o estresse no trabalho. Variáveis como afeto positivo, realização pessoal no trabalho e felicidade, de um modo geral, têm sido pouco enfatizadas na literatura organizacional, o que se observa também no campo da qualidade de vida. Paschoal, Torres e Porto (2010) demonstram a importância da inclusão de elementos com clara conotação positiva na definição de bem-estar, em oposição à tradição que o define a partir da ausência de experiências negativas. Em seu estudo sobre o impacto do suporte organizacional no bem-estar no trabalho, os autores identificaram um impacto diferenciado das variáveis independentes sobre o afeto positivo, afeto negativo e realização no trabalho. Enquanto afeto positivo e realização foram mais fortemente preditos pelo suporte voltado para a gestão do desempenho, o afeto negativo foi mais fortemente predito pelo suporte para sobrecarga de trabalho. Os achados do estudo, segundo Paschoal, Torres e Porto (2010), sustentam que o afeto negativo é mais suscetível a eventos organizacionais considerados estressores do que o afeto positivo e a realização. Os resultados sugerem ainda que afeto positivo e negativo não consistem somente em dimensões independentes do bem-estar, mas que variam com intensidades distintas mediante as mesmas características e condições laborais.

A fim de contribuir para o preenchimento dessa lacuna, o objetivo deste estudo foi investigar o impacto de atividades organizacionais voltadas à saúde do trabalhador sobre o bem-estar no trabalho. Tais atividades consistem em ações que compõem programas de qualidade de vida em organizações diversas. É preciso destacar que a QVT não se restringe a ações organizacionais voltadas à saúde do trabalhador, mas consiste em um conjunto de ações e ferramentas que visam a modificar condições de trabalho e uma visão do trabalhador em sua totalidade (Ferreira, 2006; Ferreira et al., 2009; Limongi-França, 2009). Neste estudo, porém, serão consideradas ações específicas que comumente constituem ações de QVT nas organizações.

\section{Método}

Amostra

Os participantes do estudo foram recrutados entre trabalhadores que cursavam graduação ou pós-graduação em uma faculdade localizada no Distrito Federal e colaboradores dessa mesma faculdade. Foram considerados os questionários respondidos de 130 participantes. Dentre aqueles que indicaram o sexo, 72,3\% eram mulheres e 27,7\% eram homens. A média de idade dos respondentes foi de 31 anos ( $d p=10,33$ anos) e o tempo médio de serviço na organização foi de 6,09 anos ( $\mathrm{dp}=8,92$ anos). Quanto à escolaridade, 15,4\% dos respondentes relataram ter ensino médio; $59,2 \%$ tinham superior incompleto; $15,4 \%$ possuíam superior completo; $10 \%$ haviam concluído pós-graduação.

\section{Instrumentos}

Neste estudo foi utilizado um questionário composto pela Escala de Bem-Estar no Trabalho - EBET (Paschoal \& Tamayo, 2008), questões que versavam sobre a existência de ações voltadas à saúde e ao bem-estar do trabalhador, sobre a frequência de participação em tais atividades e a percepção de contribuição das atividades para o bem-estar e o clima organizacional. A questão sobre frequência de participação apresentava escala de resposta de cinco pontos, que variava de nunca a sempre. A questão sobre a contribuição das atividades apresentava escala de resposta de quatro pontos, que variava de nada a muito. Por fim, foram incluídas questões sobre dados sócio-demográficos.

A EBET foi utilizada para mensurar a variável dependente do estudo. Esse instrumento é composto por 30 itens divididos em três fatores: 1) afeto positivo, com nove itens e índice de confiabilidade alpha de Cronbach $(\alpha)$ equivalente a 0,$95 ; 2$ ) afeto negativo, com 12 itens e $\alpha$ de 0,$94 ; 3$ ) realização, composto por nove itens e $\alpha$ igual a 0,92 . A parte de afeto no trabalho da EBET apresenta a seguinte instrução: nos últimos seis meses, meu trabalho tem me deixado. Os itens, 
como alegre, orgulhoso, animado, irritado, chateado e nervoso, devem ser respondidos de acordo com uma escala de cinco pontos, que varia de 1 (nem um pouco) a 5 (extremamente). A parte de realização no trabalho contém a instrução: neste trabalho. Itens como realizo o meu potencial, avanço nas metas que estabeleci para minha vida e expresso o que há de melhor em mim devem ser respondidos de acordo com uma escala de concordância de cinco pontos, que varia de 1 (discordo totalmente) a 5 (concordo totalmente).

\section{Procedimento}

Os questionários foram aplicados individualmente, fora do horário de aula e do expediente de trabalho. A pesquisadora explicava os objetivos do estudo, questões referentes ao sigilo e distribuía os questionários para os participantes. Os questionários eram respondidos e colocados em um envelope.

\section{Análise de dados}

Para o tratamento dos dados, foram conduzidas análises estatísticas descritivas (média, desvio-padrão, valores mínimo e máximo), análise de correlações bivariadas e regressão múltipla stepwise, que considera apenas critérios estatísticos para a entrada das variáveis no modelo de predição.

\section{Resultados}

\section{Análises descritivas}

Em relação ao afeto positivo, a média para a amostra foi de 3,09 (dp = 0,90), em uma escala de 1 a 5 . Para afeto negativo, foi encontrada média de 2,15 ( $\mathrm{dp}=$ $0,85)$; para o fator realização, foi encontrada média de 3,69 (dp = 078). A Tabela 1 apresenta esses resultados.

Tabela 1 - Resultados da análise descritiva das variáveis dependentes

\begin{tabular}{lcccc}
\hline Variável & Média & $\begin{array}{c}\text { Desvio } \\
\text { padrão }\end{array}$ & Mínimo & Máximo \\
\hline $\begin{array}{l}\text { Afeto } \\
\text { positivo }\end{array}$ & 3,09 & 0,90 & 1,00 & 5,00 \\
$\begin{array}{l}\text { Afeto } \\
\text { negativo }\end{array}$ & 2,15 & 0,85 & 1,00 & 5,00 \\
\begin{tabular}{l} 
Realização \\
\hline
\end{tabular} & 3,69 & 0,78 & 1,00 & 5,00 \\
\hline
\end{tabular}

Fonte: Dados da pesquisa.
A média mais alta de bem-estar no trabalho foi observada para a realização, cujo valor ficou acima do ponto médio da escala de resposta. Afeto negativo, que corresponde às emoções e humores negativos do trabalhador, obteve a pontuação média mais baixa.

Dos 130 participantes que responderam ao questionário, 56,2\% disseram que sua organização desenvolvia atividades voltadas à saúde e bem-estar do trabalhador. Os tipos de atividades desenvolvidas pelas organizações encontram-se descritas na Tabela 2. Como é possível observar, as atividades mais frequentes são as comemorações de aniversariantes, que expressam eventos de integração social, e a ginástica laboral.

Tabela 2 - Atividades voltadas à saúde do trabalhador

\begin{tabular}{lcc}
\hline Atividades & Frequência & Porcentagem (\%) \\
\hline Ginástica laboral & \multicolumn{1}{l}{} \\
Sim & 46 & 35,4 \\
Não & 84 & 64,6 \\
\hline Momentos de relaxamento & \\
Sim & 14 & 10,8 \\
Não & 116 & 89,2 \\
Sala com musicalização & & \\
Sim & 10 & 7,7 \\
Não & 120 & 92,3 \\
Comemoração dos aniversariantes & \\
Sim & 89 & 68,5 \\
Não & 41 & 31,5 \\
Automassagem & & \\
Sim & 21 & 16,2 \\
Não & 109 & 83,8 \\
\hline
\end{tabular}

Fonte: Dados da pesquisa.

\section{Correlação}

Conforme a Tabela 3, houve correlação significativa entre a frequência de participação em atividades de saúde, a percepção da contribuição das ações e afeto negativo. Todas as correlações foram negativas. Isso significa que quanto mais as pessoas participam de atividades voltadas para a saúde do trabalhador e melhor é a percepção da contribuição das ações, menor é o afeto negativo que elas vivenciam. 
Tabela 3 - Correlacões entre variáveis do estudo

\begin{tabular}{lccccc}
\hline & 1 & 2 & 3 & 4 & 5 \\
\hline $\begin{array}{l}\text { 1. Afeto positivo } \\
\text { 2. Afeto negativo }\end{array}$ & $-0,59^{* *}$ & & & & \\
\hline 3. Realização & $0,61^{* *}$ & $-0,27^{* *}$ & & & \\
\hline $\begin{array}{l}\text { 5. Frequência de } \\
\text { participação em } \\
\text { atividade de saúde }\end{array}$ & $-0,01$ & $-0,34^{*}$ & $-0,05$ & & \\
$\begin{array}{l}\text { 6. Percepção da } \\
\text { contribuição para o } \\
\text { bem-estar }\end{array}$ & 0,01 & $-0,21^{*}$ & 0,05 & $0,50^{* *}$ & \\
$\begin{array}{l}\text { 7. Percepção da } \\
\text { contribuição das } \\
\text { ações para clima }\end{array}$ & 0,03 & $-0,28^{* *}$ & 0,13 & $0,62^{* *}$ & $0,67^{* *}$ \\
\hline
\end{tabular}

Legenda: ${ }^{*} \mathrm{p}<0,05$

$$
{ }^{* *} \mathrm{p}<0,01
$$

Fonte: Dados da pesquisa.

\section{Regressão}

Foram conduzidas regressões do tipo stepwise para cada variável dependente, a fim de testar o efeito preditivo da participação em atividades de saúde, da frequência da participação e da percepção da contribuição das ações sobre afeto positivo, afeto negativo e realização. Afeto positivo e realização não sofreram influência de qualquer variável independente. Participar das atividades voltadas à saúde, participar com alta frequência e perceber positivamente a contribuição das ações voltadas à saúde são variáveis que não modificam as experiências positivas do bem-estar. A única relação significativa observada foi entre frequência de participação nas atividades voltadas à saúde e afeto negativo. Os resultados das regressões revelaram que a frequência de participação em atividades explica 10\% da variância do afeto negativo. $\mathrm{O}$ valor do $\mathrm{R}$ foi de 0,34 e do $\mathrm{R}^{2}$ ajustado foi de 0,10 . 0 valor de $B$ foi de $-0,17$ e o do $\beta$ foi de $-0,34$ ( $p<0,001)$. Quanto maior é a frequência de participação em ações voltadas para a saúde do trabalhador, menor é a experiência de afeto negativo.

\section{Discussão}

Este trabalho teve como objetivo principal verificar o impacto de atividades organizacionais voltadas à saúde sobre o bem-estar do trabalhador. 0 desafio que aproxima pesquisadores de qualidade de vida e bem-estar reside na tentativa de introduzir, no campo da teorização e nas práticas psicológicas organizacionais, uma concepção diferenciada, focada na mudança e na transformação de processos comportamentais.

As análises descritivas dos itens sobre ações voltadas à saúde e bem-estar do trabalhador indicam que as atividades mais frequentemente desenvolvidas pela organização se referem a eventos sociais, como a comemoração de aniversários, e à ginástica laboral. Quanto ao bem-estar no trabalho, os resultados sugerem que existe bem-estar na percepção dos participantes, visto que os valores para afeto positivo e realização encontram-se acima do ponto médio da escala de resposta. Por outro lado, a média para afeto negativo também foi muito próxima do ponto médio da escala, indicando que esta é uma experiência significativa dos trabalhadores participantes.

Quanto ao impacto das ações voltadas à saúde, as correlações indicaram apenas associações significativas com o afeto negativo. Na regressão, a única variável que explicou o bem-estar foi a frequência de participação nas atividades, que influenciou negativamente o afeto negativo. Mais do que a existência de atividades voltadas à saúde, a frequência da participação nas atividades é importante para diminuir o afeto negativo. Quanto mais frequente é a participação do trabalhador em atividades como ginástica laboral, momentos de relaxamento, sala com musicalização, comemoração dos aniversariantes e automassagem, menos frequentes e intensas são suas experiências afetivas negativas. Tal resultado sugere que esse tipo de atividade tem relevância para o afeto negativo no trabalho e os gestores devem estar atentos a tais práticas.

Afeto positivo e realização não sofreram nenhuma influência dessas variáveis. Tal resultado vai ao encontro do estudo de Paschoal et al. (2010), que verificou que os elementos positivos do bem-estar têm preditores diferentes daqueles relacionados aos elementos negativos. A felicidade ou realização pessoal não é predita pelas mesmas variáveis que influenciam o estresse e o afeto negativo. As ações voltadas para a saúde aqui investigadas minimizam emoções negativas, típicas de experiências estressantes, mas não favorecem o prazer no trabalho e a realização e expressão pessoal do trabalhador.

Segundo Ferreira (2006) e Ferreira et al. (2009), as ações abordadas neste estudo são típicas de uma 
abordagem assistencialista de QVT. Os referidos autores já alertaram para o caráter paliativo de tais atividades. Condições do ambiente influenciam o bem-estar e os comportamentos do trabalhador, e os programas de QVT buscam aliviar os sintomas negativos vivenciados no dia a dia organizacional. Os resultados do presente estudo oferecem suporte às observações de Ferreira (2006) e Ferreira et al. (2009), indicando que experiências mais positivas do bem-estar não são influenciadas pelas ações assistencialistas.

Muitos programas voltados à QVT enfocam ações assistencialistas e, muitas vezes, restringem-se a elas. Segundo Limongi-França (2009), os gestores devem identificar os componentes psicossociais da organização, entendendo a importância do significado do trabalho, dos treinamentos e projetos de educação para o trabalho e das novas tecnologias. Segundo a autora, QVT se relaciona à produtividade, à legitimidade, à responsabilidade social, ao desenvolvimento sustentável, à cultura organizacional, à competitividade e aos valores organizacionais. As ações relacionadas à QVT, portanto, envolvem uma ampla gama de práticas e políticas organizacionais.

Quando as ações propostas pelos gestores buscam minimizar o afeto negativo, as atividades descritas neste estudo podem contribuir. Se os gestores realmente pretendem contribuir para o desenvolvimento, realização pessoal e o prazer no trabalho, outras ações devem ser pensadas. Por exemplo, aquelas atividades que aumentem participação do trabalhador em decisões, autonomia e investimento em educação podem representar estratégias importantes (Paschoal et al., 2010; Warr, 2007). Para Limongi-França (2009), é importante que os gestores entendam que ações de QVT são investimentos da organização e não uma ação filantrópica. Estudos sugerem que os gestores consideram QVT um tema estratégico para o aumento da produtividade em ambientes competitivos, mas ainda carecem de maiores informações sobre a amplitude e complexidade dos programas.

Apesar de ter contribuído para preencher lacunas referentes ao campo do bem-estar no trabalho, o presente estudo apresenta limitações. Primeiramente, as ações de qualidade de vida ou voltadas para a saúde abordadas no presente estudo não representam toda a variedade de atividades que compõem os programas de QVT nas organizações. Estudos futuros deverão investigar e descrever as ações de QVT mais comumente desenvolvidas pelas organizações públicas e privadas no Brasil. Posteriormente, os efeitos de cada uma sobre os fatores de bem-estar deverão ser testados. Outro aspecto importante do campo do bem-estar laboral é que, mesmo quando se investiga o impacto de condições de trabalho e práticas organizacionais sobre o bem-estar, os pesquisadores consideram apenas o nível individual de análise. Deve-se investigar se as práticas e ações de QVT, avaliadas no nível da organização ou dos grupos, também influenciam as dimensões de bem-estar. Para tanto, técnicas de coleta e análise de dados multiníveis deverão ser consideradas.

\section{Referências}

Albuquerque, A. S., \& Tróccoli, B. T. (2004). Desenvolvimento de uma escala de bem-estar subjetivo. Psicologia: Teoria e Pesquisa, 20(2), 153-164.

Barsky, A., \& Kaplan, S. A. (2007). If you feel bad, it's unfair: A quantitative synthesis of affect and organizational justice perceptions. Journal of Applied Psychology, 92(1), 286-295.

Carvalho, I. V., Leite, L. A. M., Rohm, R. H. D., \& Oliveira, J. L. C. (2008). Consultoria em gestão de pessoas. Rio de Janeiro: FGV.

Diener, E., \& Suh, E. M. (1999). National differences in subjective well-being. In D. Kahneman, E. Diener \& N. Schwarz (Org.). Well-being: The foundations of hedonic psychology (pp. 334-350). New York: Russel Sage Foundations.

Ferreira, M. C. (2006). Qualidade de vida no trabalho (QVT). In A. D. Cattani \& L. Holzmann (Org.). Dicionário de trabalho e tecnologia (pp. 219-222). Porto Alegre: Ed. da UFRGS.

Ferreira, R. R., Ferreira, M. C., Antloga, C. S., \& Bergamaschi, V. (2009). Concepção e implantação de um programa de qualidade de vida no trabalho no setor público: O papel estratégico dos gestores. Revista de Administração, 44(2), 147-157.

Jex, S. M., \& Spector, P. E. (1996). The impact of negative affectivity on stressor-strain relations: A replication and extension. Work and Stress, 10(1), 36-45. 
Limongi-França, A. C. (1997). Qualidade de vida no trabalho: Conceitos, abordagens, inovações e desafios nas empresas brasileiras. Revista Brasileira de Medicina Psicossomática, 1(2), 79-83.

Limongi-França, A. C. (2009). Qualidade de vida no trabalho - QVT: Conceitos e práticas nas empresas da sociedade pós-industrial. São Paulo: Atlas.

Paschoal, T., \& Tamayo, A. (2008). Construção e validação da escala de bem-estar no trabalho. Avaliação Psicológica, 7(1), 11-22.

Paschoal, T., Torres, C. V., \& Porto, J. B. (2010). Felicidade no trabalho: Relações com suporte organizacional e suporte social. Revista de Administração Contemporânea, 14(6), 1054-1072.

Ryff, C. D. (1989). Happiness is everything, or is it? Explorations on the meaning of psychological well-being. Journal of Personality and Social Psychology, 57(6), 1069-1081.

Strazdins, L., D’Souza, R. M., Lim, L. L. Y, Broom, D. H., \& Rodgers, B. (2004). Job strain, job insecurity, and health: Rethinking the relationship. Journal of Occupational Health Psychology, 9(4), 296-305.
Vasconcelos, A. F. (2001). Qualidade de vida no trabalho: Origem, evolução e perspectivas. Caderno de Pesquisas em Administração, 8(1), 23-32.

Warr, P. (2007). Work, happiness and unhappiness. New Jersey: Lawrence Erlbaum Associates.

Waterman, A. S. (1993). Two conceptions of happiness: Contrasts of personal expressiveness (Eudaimonia) and hedonic enjoyment. Journal of Personality and Social Psychology, 64(4), 678-691.

Waterman, A. S., Schwartz, S. J., \& Conti, R. (2008). The implications of two conceptions of happiness (hedonic enjoyment and eudaimonia) for the understanding of intrinsic motivation. Journal of Happiness Studies, 9(1), 41-79.

Weiss, H. M., Suckow, K., \& Cropanzano, R. (1999). Effects of justice conditions on discrete emotions. Journal of Applied Psychology, 84(5), 786-794.

Wiese, B. S., \& Freund, A. M. (2005). Goal progress make one happy, or does it? Longitudinal findings from the work domain. Journal of Occupational and Organizational Psychology, 78(2), 287-304. 